\title{
Care Among Migraine Patients in a Commercially Insured Population
}

\author{
Machaon Bonafede · Donna McMorrow • Virginia Noxon • \\ Pooja Desai $\cdot$ Sandhya Sapra $\cdot$ Stephen Silberstein
}

Received: November 26, 2019 / Published online: February 15, 2020

(C) The Author(s) 2020

\begin{abstract}
Introduction: Migraine management is characterized by the poor use of preventive therapy and the overuse of acute medications. An analysis of current treatment patterns in migraineurs is needed to improve care in this patient population. The aim of this study was to describe treatment patterns and healthcare utilization of newly diagnosed migraine patients.

Methods: This was a retrospective observation study of newly diagnosed migraine patients (no indication of migraine in the past year) identified in the IBM MarketScan Commercial Claims and Encounters database from 1 January 2010 to 30 June 2014 . The final study population comprised persons aged 18-64 years at index (new diagnosis of migraine) with 12 months of continuous enrollment in an insurance plan with medical and pharmacy benefits pre-index and post-index. Treatment patterns and healthcare
\end{abstract}

Enhanced Digital Features To view enhanced digital features for this article go to https://doi.org/10.6084/ m9.figshare.11710158.

M. Bonafede $(\varangle) \cdot$ D. McMorrow $\cdot$ V. Noxon IBM Watson Health, Cambridge, MA, USA

e-mail: mbonafed@us.ibm.com

P. Desai · S. Sapra

Amgen, Thousand Oaks, CA, USA

S. Silberstein

Jefferson Medical College, Philadelphia, PA, USA resource utilization were assessed during the post-index period (at least 12-months).

Results: Of the 1,588,666 migraine patients identified in the database as potentially eligible to participate in the study, 284,719 (17.9\%) met the final inclusion criteria. Patients generally used acute and preventive therapies to manage migraine attacks, with most patients using preventive therapy $(59.1 \%)$. However, $67.9 \%$ of those using preventive therapy discontinued the current therapy, with a median time to discontinuation of 5 months. Most of the patients who discontinued preventative therapy also used an acute treatment to manage migraine attacks after discontinuation (77.6\%), generally in the year following discontinuation $(68.4 \%)$. Patients on acute therapies were found to use triptans excessively (1.6\%) and other non-migraine-specific acute medications for treatment $(7.1 \%)$. Acute patients were also at risk of opioid dependence (12.0\%) and commonly received opioids or barbiturates as firstline therapy (34.1\%).

Conclusion: Newly diagnosed migraine patients are not being properly treated, as indicated by their excessive use of acute therapies and short time on preventive treatment before discontinuation of that treatment. Further study of the reasons why patients discontinue preventive therapy (adverse events, no response, etc.) and continue to excessively use acute treatments once their treatment regimen has been established is needed. 
Keywords: Acute medication; Healthcare utilization; Migraine; Preventive medication

\section{Key Summary Points}

Why carry out this study?

Only a fraction of those suffering from migraine receive proper care with preventive or acute treatment despite the diminished quality of life due to migraines.

Treatment improves the quality of life of migraine patients; therefore, the aim of this study was to explore treatment patterns among newly diagnosed migraine patients in a real-world setting to add to the existing literature on the treatment of and care received by migraine patients.

\section{What was learned from the study?}

Of the newly diagnosed migraine patients included in the analysis, $59.1 \%$ used preventive treatment; $67.9 \%$ of these patients discontinued their preventive treatment within 5.4 months and then used an acute treatment (77.6\%) or arranged a neurology consult (18.3\%).

Newly diagnosed migraine patients are high utilizers of migraine healthcare services, particularly acute treatments.

The results highlight a potential correlation between poor treatment management and increased migrainespecific healthcare utilization.

\section{INTRODUCTION}

Migraine attacks have at least two of the following characteristics (1) a moderate to severe intensity; (2) unilateral (one side of the head) location; (3) presence of throbbing; and (4) worsening with increased physical activity [1]. The International Headache Society defines a migraine as when a person reports five or more attacks of headache that each last between 4 and $72 \mathrm{~h}$, with the attacks meeting at least two of the above four criteria and being associated with either (1) nausea and/or vomiting or (2) photophobia and phonophobia [1, 2].

Migraine attacks can be treated acutely or preventively depending on individual need. Acute treatment is given at the onset of a migraine headache to reduce pain and the associated symptoms and generally consists of triptans, ergotamines/dihydroergotamine, opioids or barbiturates [3]. In contrast, preventive treatment is taken on a regular basis with the aim to decrease the frequency and intensity of migraine attacks as well as the related disability; such treatment consists of select anticonvulsants, beta-blockers, calcium channel blockers and tricyclic antidepressant drugs [3, 4]. The ultimate goal of preventive treatment is to reduce excessive use of acute treatments $[3,4]$. Calcitonin gene-related peptide inhibitors are monoclonal antibodies (e.g., erenumab, fremanezumab and galcanezumab) and relatively new preventive therapies for migraine that were not included in the studies mentioned above as they had not been commercially available at the time $[5,6]$. Migraine attacks are undertreated, particularly in terms of preventive treatment. A study of treatment patterns for migraine attacks estimated that while $38.8 \%$ of migraine patients were candidates for preventive migraine treatment, only $12.4 \%$ actually received preventive treatment, a frequency that reflects underutilization of preventive treatment [7].

Those persons affected by migraine have a diminished quality of life, including work time missed, increased healthcare utilization, such as increased number of visits to the emergency room (ER) and increased healthcare costs $[8,9]$. However, should such persons be properly treated, both their health and quality of life would be improved and healthcare resource utilization (HRU) and costs would be reduced $[8,9]$. A study of migraine patients in a tertiary headache center reported that those who used both acute and preventive treatment regimens had a marked reduction in the Migraine Disability Assessment Score (MIDAS) after starting treatment, indicating an improvement in their quality of life [10]. This improvement in quality 
of life was markedly better for those identified as overusing acute medication prior to starting their new treatment, which was a properly controlled acute and prophylactic regimen. This study also reported a marked reduction in healthcare utilization and cost after the new combined treatment was started, particularly 6-month cost, office visits and ER visits [10]. Another study of migraine patients with employer-sponsored health insurance reported that approximately $45 \%$ of diagnosed migraine patients did not receive proper treatment, but those who did have proper treatment had significantly reduced all-cause healthcare cost, migraine-related inpatient (IP) admissions, ER visits and office visits [11].

The literature highlights poor use of migraine therapy (poor preventive therapy use and overuse of acute therapies) and shows that when properly treated, migraine patients have an improved quality of life that includes reduced healthcare costs and HRU.

Currently, there are two migraine registries (AXON and American Registry for Migraine Research) that collect patient-reported data on migraine that includes treatment and additional quality of life measures $[12,13]$. Even as these registries become a primary source of information on migraine outcomes and treatment, there is still limited information available on real-world outcomes (i.e. HRU, acute and preventive treatment use) and quality of care for newly diagnosed migraine patients $[12,13]$. In this context, the aim of this study was to expand the body of information currently available on healthcare utilization by migraine patients and on the treatment received by these patients by evaluating potential quality of care indicators for newly diagnosed migraine patients with a focus on treatment (preventive and acute) and HRU in a commercially insured claims dataset.

\section{METHODS}

\section{Data Sources}

Data for analysis in this study were retrieved from the IBM MarketScan ${ }^{\circledR}$ Commercial
Database and covered the period from 1 January 2009 to 30 June 2015. This database contains administrative claims for over 132 million employees and their dependents with differing healthcare plans in the USA.

This article is based on previously conducted studies and does not contain any studies with human participants or animals performed by any of the authors. All database records are statistically de-identified and certified to be fully compliant with US patient confidentiality requirements set forth in the Health Insurance Portability and Accountability Act of 1996. Because this study used only de-identified patient records and did not involve the collection, use or transmittal of individually identifiable data, Institutional Review Board approval to conduct this study was not required.

\section{Cohort Selection}

Those patients who met at least one the following criteria from 1 January 2010 through to 30 June 2014 were included in the initial migraine cohort: (1) at least one IP admission with a diagnosis of migraine (International Classification of Diseases, Ninth Revision, Clinical Modification [ICD-9-CM] 346.xx; https://www.cdc.gov/nchs/icd/icd9cm.htm) in any position on the claim; (2) at least two ER claims (7-180 days apart) with a diagnosis of migraine in any position on the claim; (3) at least two outpatient claims with a diagnosis of migraine (7-180 days apart) in any position on the claim; (4) at least one outpatient claim with a diagnosis of migraine in any position on the claim and at least one outpatient pharmacy claim for a triptan or ergotamine/dihydroergotamine (acute migraine medications) or topiramate (prophylactic migraine medication) (7-180 days apart); (5) at least one outpatient neurologist visit with a diagnosis of migraine in any position; (6) at least two outpatient pharmacy claims for a triptan or ergotamine/dihydroergotamine (7-180 days apart) (i.e. two triptan claims, two ergotamine claims or one triptan and one ergotamine claim). The date of first diagnosis was defined as the index date. Those patients who qualified based on two 
claims had their index date set to the first of the two claims as the index date was set after final inclusion into the sample population.

The final migraine population consisted of patients who were 18-64 years of age on the index date, were continuously enrolled in medical and pharmacy benefits for at least 12 months before and after the index date and showed no evidence of migraine observed in the year prior to the index date.

\section{Study Covariates}

All demographic variables were measured on the index date. Clinical characteristics, including the Deyo Charlson-Comorbidity Index (DCI), were measured during the 12 months prior to the index date. The DCI is a measure of general health status that is adapted for administrative claims data through its use of ICD-9 diagnosis codes to identify the different disease states that comprise the DCI [14].

\section{Outcomes}

All reported outcomes were measured during the variable-length follow-up period, which was defined as the time from the index date until the earliest of IP death, end of continuous enrollment or 30 June 2015 (end of study).

The following measures of acute migraine treatment were described: patients with any use, who also used preventive treatment, had an opioid or barbiturate as first-line acute treatment and showed excessive use of acute treatment. Excessive use of acute medication was described as the use of migraine-specific ( $\geq 10$ pills of triptans per month) or migraine nonspecific $[\geq 15$ pills per month of non-steroidal anti-inflammatory drugs (NSAIDs) or 10 pills of opioids or barbiturates per month] medications $[1,15]$.

The following measures were described among patients initiating up to three lines of preventative treatments: time to first preventive treatment from index; duration of each preventive treatment; and time between preventive treatments. Duration of preventive treatment was defined as the time from the start of a specific preventive treatment to whichever of the following was earliest: (1) start of new preventive treatment; (2) discontinuation of the current preventive treatment; (3) end of followup. Discontinuation was defined as a gap of at least 60 days in treatment after the daily supply of the last prescription was exhausted. Time between preventive treatments was defined as the time from the end of the prior preventive treatment (including daily supply of last filled prescription) to the start of the next treatment. Additional measures among patients who discontinued preventive treatment included those who used acute medication after discontinuing preventive treatment at any time after discontinuation and within the first year, those who restarted preventive treatment after discontinuing, and time from discontinuation to end of follow-up.

All-cause and migraine-specific HRU was described for IP admissions, ER visits and physician office visits. Migraine-specific utilization was defined as any IP admission, ER visit or physician office visit for migraine (ICD-9 code 346.xx). Additional measures of HRU included patients with a migraine-related physician office visit or neurologist after a migraine-related ER visit (anytime and within 14 days).

\section{Data Analysis}

The mean and standard deviation were reported for all continuous variables while frequencies and percentages were reported for categorical variables. Medians were reported on select continuous variables. Continuous measures were reported as per patient per month (PPPM) to account for the variable-length follow-up period. Descriptive results are presented among all migraine patients unless otherwise indicated. Data extraction was performed using SAS version 9.4 (SAS Institute Inc., Cary, NC, USA).

\section{RESULTS}

The final study sample consisted of 284,719 patients with migraine who met all of the study inclusion criteria (Fig. 1). The average age of the patients was $40.1 \pm 12.1$ years and $79.1 \%$ were 


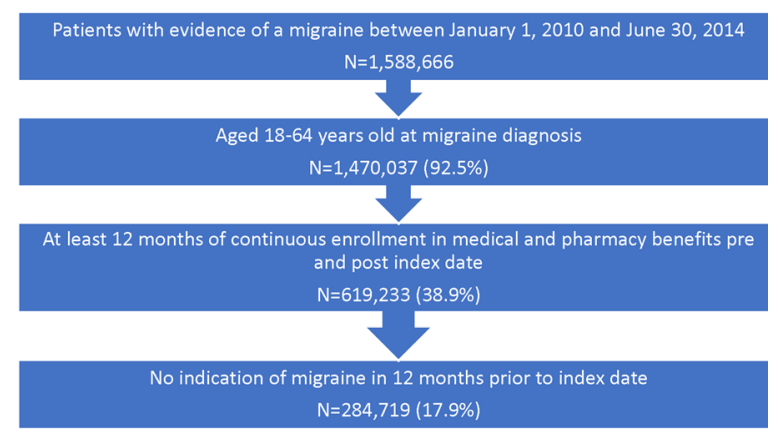

Fig. 1 Flow chart of patient selection

women; the average duration of follow-up was $31.3 \pm 14.2$ months (Table 1 ). Commonly observed baseline comorbid conditions (> 10\%) were headache $(28.5 \%)$, chronic pain $(22.3 \%)$, hypertension (17.2\%), depression (12.8\%), chest pain (11.3\%) and anxiety (11.0\%). Patients had an average baseline DCI of $0.3 \pm 0.8$.

All-cause and migraine-related healthcare utilization by the study population during follow-up was analyzed. During follow-up, patients generally visited a physician's office (99.5\%; referred to as office visits henceforth) or the ER (49.5\%) for any reason (Table 2 ). Patients also generally used the following healthcare services for migraine: office visits (83.3\%), neurologist visits (38.5\%), ER visits (11.6\%) and neuroimaging scans (11.5\%) (Table 2).

Office visits, both all-cause $(20.0 \pm 18.3$ visits PPPM) and migraine-specific ( $2.7 \pm 3.7$ visits PPPM), were the most utilized healthcare resource among the study patients, followed by neurologist visits $(1.0 \pm 2.2$ visits PPPM) and neuroimaging scans $(0.1 \pm 0.4$ scans PPPM $)$ (Table 2). The ER was not regularly utilized for any reason $(1.4 \pm 3.5$ visits PPPM $)$ or for migraine $(0.2 \pm 0.9$ visits PPPM). In contrast, those with at least one migraine-related ER visit averaged a markedly higher number of visits $(1.6 \pm 2.1$ visits PPPM). Following a migrainespecific ER visit, migraine-specific office (8.1\%) and neurologist $(3.9 \%)$ visits were not commonly observed. Of those who had a migrainerelated office/neurologist visit after a migrainerelated ER visit, less than half made an office $(36.5 \%)$ or neurologist (30.2\%) visit within 2 weeks; on average, these visits occurred >
Table 1 Patient characteristics

\begin{tabular}{ll}
\hline Patient characteristics & $\begin{array}{l}\text { All study patients } \\
(\boldsymbol{N}=\mathbf{2 8 4 , 7 1 9 )}\end{array}$ \\
\hline Age (years) & $40.1 \pm 12.1$ \\
Female & $225,213(79.1 \%)$ \\
Index year & \\
2010 & $74,807(26.3 \%)$ \\
2011 & $71,804(25.2 \%)$ \\
2012 & $62,411(21.9 \%)$ \\
2013 & $56,729(19.9 \%)$ \\
2014 & $18,968(6.7 \%)$ \\
Length of follow-up & $31.3 \pm 14.2$ \\
(months) & \\
Baseline DCI & $0.3 \pm 0.8$ \\
Baseline comorbid conditions \\
Anxiety & $31,340(11.0 \%)$ \\
Chest pain & $32,168(11.3 \%)$ \\
Chronic pain & $63,379(22.3 \%)$ \\
Depression & $36,573(12.8 \%)$ \\
Headache & $81,143(28.5 \%)$ \\
Hypertension & $48,878(17.2 \%)$ \\
Treatment utilization & \\
No treatment & $22,730(8.0 \%)$ \\
Acute only & $93,848(32.9 \%)$ \\
Acute and preventive & $155,386(54.6 \%)$ \\
Preventive only & $12,755(4.5 \%)$ \\
\hline Valss are giva & \\
\hline
\end{tabular}

Values are given as the mean \pm standard deviation (SD) or as a number $(n)$ with the percentage in parenthesis DCI Deyo-Charlson Comorbidity Index

100 days after a migraine-related ER visit (office visits $111.0 \pm 206.2$ days; neurologist visits $132.7 \pm 236.9$ days). The smallest category of observed healthcare utilization was IP admissions for any reason (20.4\%) and for migraine (6.5\%) (Table 2). 
Table 2 All-cause and migraine-related healthcare utilization during follow-up

\begin{tabular}{ll}
\hline Healthcare utilization & $\begin{array}{l}\text { All study patients } \\
(\boldsymbol{N}=\mathbf{2 8 4 , 7 1 9 )}\end{array}$ \\
\hline All-cause & \\
Inpatient admission & $58,173(20.4 \%)$ \\
PPPM admissions & $0.3 \pm 1.0$ \\
ER visit & $140,870(49.5 \%)$ \\
PPPM visits & $1.4 \pm 3.5$ \\
Outpatient office visit & $283,391(99.5 \%)$ \\
PPPM visits & $20.0 \pm 18.3$ \\
Migraine-related & \\
Inpatient admission & $18,536(6.5 \%)$ \\
PPPM admissions & $0.1 \pm 0.3$ \\
ER visit & $32,957(11.6 \%)$ \\
PPPM visits & $0.2 \pm 0.9$ \\
Outpatient office visit & $237,078(83.3 \%)$ \\
PPPM visit & $2.7 \pm 3.7$ \\
Neurologist & $109,623(38.5 \%)$ \\
PPPM visit & $1.0 \pm 2.2$ \\
Neuroimaging scan & $32,657(11.5 \%)$ \\
PPPM scans & $0.1 \pm 0.4$ \\
Office visit after migraine- & $22,988(8.1 \%)$ \\
related ER visit & \\
Within 2-weeks & $8379(36.5 \%)$ \\
Neurologist visit after migraine- & $10,967(3.9 \%)$ \\
related ER visit & \\
Within 2-weeks & $3314(30.2 \%)$ \\
\hline V & $\pm 5 D$ \\
\hline
\end{tabular}

Values are given as the mean $\pm S D$ or as a number (n) with the percentage in parenthesis

$E R$ Emergency room, PPPM per patient per month

In general, patients used both acute and preventive therapies $(54.6 \%)$ or only acute therapy (32.9\%) (Table 1). More than half of the study patients used preventive medication (59.1\%) and started treatment within
0.7 months (median) of their diagnosis. Most patients used their first preventive treatment for a median of 4.0 months before discontinuing treatment or switching to another treatment. Of note, the median time to discontinuation of the first preventive treatment was 5.4 months (Table 3).

Only one-half of those who started a first preventive treatment $(48.5 \%)$ went onto a second preventive treatment, with $50 \%$ of the latter starting the second preventive treatment within 2.6 months of ending their first preventive treatment. Patients starting on a second preventive treatment stayed on that treatment for a median of 3.0 months, and $45.0 \%$ subsequently started a third preventive treatment. The median time from the end of the second preventive treatment to the start of the third preventive treatment was 2.9 months, and the median duration of the third preventive treatment was 2.7 months (Table 3). About one-half $(48.8 \%)$ of patients who discontinued preventive therapy never started another preventive therapy during the median post-discontinuation follow-up time of 26.5 months. Among the discontinuers of preventive therapy, $77.6 \%$ started on an acute therapy (43.5\% within 3 months of preventive therapy discontinuation; $68.4 \%$ within 1 year), $18.3 \%$ visited a neurologist and $6.2 \%$ had a migraine-specific ER visit in the post-discontinuation follow-up period (Table 3).

In general, all patients on acute medication used opioids $(68.3 \%)$, triptans $(63.0 \%)$ or prescription NSAIDs (46.6\%) (Table 4); in comparison, among those only on acute therapy, fewer patients used opioids (58.2\%) and prescription NSAIDs (40.5\%). Triptan use was similar among all users of acute therapy (63.0\%) and users of acute therapy only $(64.3 \%)$. The use of opioids or barbiturates as the first acute migraine therapy was observed in $34.1 \%$ of all acute therapy users and in $29.5 \%$ of acute therapy-only users. An excessive use of triptans was observed in $1.6 \%$ of all acute therapy users and in $1.3 \%$ of acute therapy-only users. The excessive use of prescription NSAIDs, opioids and barbiturates based on ICHD-3b (https://www.ihs-headache. org/ichd-guidelines) criteria was observed in $7.1 \%$ of all acute therapy users and in $4.1 \%$ of 
Table 3 Preventive treatment measures used by patients during follow-up

\begin{tabular}{ll}
\hline Preventive treatment measures & All study patients $(\boldsymbol{N}=\mathbf{2 8 4 , 7 1 9 )}$ \\
\hline Initiate preventive treatment & $168,141(59.1 \%)$ \\
Median time to first treatment (months) & 0.7 \\
Median length of first treatment (months) & 4.0 \\
Initiate second preventive treatment & $81,563(48.5 \%)$ \\
Median time to second treatment (months) & 2.6 \\
Median length of second treatment (months) & 3.0 \\
Initiate third preventive treatment & $36,693(45.0 \%)$ \\
Median time to third treatment (months) & 2.9 \\
Median length of third treatment (months) & 2.7 \\
Discontinue preventative treatment & $114,265(67.9 \%)$ \\
Median time to discontinuation (months) & 5.4 \\
Post-discontinuation measures & \\
Restart preventative treatment & $58,474(51.2 \%)$ \\
Use acute treatment & $88,682(77.6 \%)$ \\
Have a migraine-specific neurological visit & $20,933(18.3 \%)$ \\
Have a migraine-specific ER visit & $7048(6.2 \%)$ \\
Median follow-up time post-discontinuation (months) & 26.5 \\
\hline
\end{tabular}

Values are given as a number $(n)$ with the percentage in parenthesis, unless indicated otherwise

acute therapy-only users. When a less strict definition of excessive use ( $\geq 10$ pills a month of prescription NSAIDs, opioids or barbiturates) was applied, $23.6 \%$ of all acute therapy users and $12.4 \%$ of acute therapy-only users were defined as showing excessive use of medications. Although an excessive use of acute medication was found in $<10 \%$ of all patients $(7.1 \%$ of all patients on acute medication and $4.1 \%$ of those only on acute medications), $12.0 \%$ of all acute therapy users and $4.7 \%$ of acute therapyonly users still had $>5$ days of opioid supply per month over a 12-month period (Table 4).

\section{DISCUSSION}

The results of this study describe patterns of healthcare utilization and treatment among newly diagnosed migraine patients in a commercially insured dataset. Consistent with previously published migraine cohorts, the study population was predominantly female who were approximately 40 years old $[9,11]$. The use of acute and preventive treatment was higher in our patient population than reported previously [9], but the patient population in this prior study comprised of established migraine patients whereas only newly diagnosed migraine patients were enrolled in our study. Migraine management requires time, and a higher use of both preventive and acute treatments in newly diagnosed migraine patients within the first years of diagnosis is expected [16, 17]. Although the utilization measures reported here are generally consistent with those in the literature, our results are slightly higher than those previously reported. Observed utilization differences could be due to differences in migraine severity, differing time 
Table 4 Acute treatment use during follow-up

\begin{tabular}{|c|c|c|}
\hline Acute treatment use & $\begin{array}{l}\text { All users of acute medications } \\
(N=249,234)\end{array}$ & $\begin{array}{l}\text { Users of acute medications } \\
\text { only }(N=93,848)\end{array}$ \\
\hline \multicolumn{3}{|l|}{ Use of acute treatments } \\
\hline Barbiturates & $44,560(17.9 \%)$ & $10,927(11.6 \%)$ \\
\hline Muscle relaxants & $90,948(36.5 \%)$ & $23,459(25.0 \%)$ \\
\hline Neuroleptics & $58,497(23.5 \%)$ & $14,914(15.9 \%)$ \\
\hline Opioids & $170,250(68.3 \%)$ & $54,635(58.2 \%)$ \\
\hline Prescription NSAIDs & $116,191(46.6 \%)$ & $38,000(40.5 \%)$ \\
\hline Triptans & $156,918(63.0 \%)$ & $60,313(64.3 \%)$ \\
\hline \multicolumn{3}{|l|}{ Measures of excessive use } \\
\hline $\begin{array}{l}\text { Treated with opioids or barbiturates as first-line } \\
\text { therapy }\end{array}$ & $85,013(34.1 \%)$ & $27,715(29.5 \%)$ \\
\hline Excessive use of triptans, based on ICHD- $3 b^{a}$ & $3983(1.6 \%)$ & $1243(1.3 \%)$ \\
\hline $\begin{array}{l}\text { Excessive use of non-migraine specific acute } \\
\text { medications, based on ICHD- } 3 b^{b}\end{array}$ & $17,581(7.1 \%)$ & $3824(4.1 \%)$ \\
\hline $\begin{array}{l}\text { Excessive use of non-migraine specific acute } \\
{\text { medications, alternate } \text { criteria }^{c}}^{c}\end{array}$ & $58,820(23.6 \%)$ & $11,628(12.4 \%)$ \\
\hline Have an opioid day supply of $>5$ days per month & $29,967(12.0 \%)$ & $4449(4.7 \%)$ \\
\hline
\end{tabular}

All values are given as a number $(n)$ with the percentage in parenthesis

ICHD-3b The International Classification of Headache Disorders 3rd edition (beta version), NSAIDs non-steroidal antiinflammatory drugs

${ }^{a}$ Based on ICHD-3b, defined as $\geq 10$ pills of triptan per month

b Based on ICHD-3b, defined as $\geq 15$ pills a month of prescription NSAIDs or $\geq 10$ pills a month of opioids or barbiturates

${ }^{c}$ Defined as $\geq 10$ pills a month of prescription NSAIDs, opioids or barbiturates

periods observed and data used; in addition, newly diagnosed patients may use more healthcare resources than established patients to obtain a treatment regimen [11, 18, 19]. Future work in administrative claims should be limited to direct comparisons of utilization between newly diagnosed and established migraine patients as migraine severity cannot be measured in administrative claims.

The reason underlying the high discontinuation rate of preventive treatments (both the initial and second treatment) could not be determined in this study, but the high discontinuation rate itself may indicate poor migraine management. As we did not determine migraine severity, the specific preventive treatment that was started, or adverse events, further study is needed to determine if the high discontinuation rate was due to poor migraine management, successful treatment and therefore no requirement for additional treatment or another reason. Non-successful reasons include patients who settled on acute treatment alone (i.e. those with less severe migraine attacks) or those who had to switch from their initial preventive treatment (i.e. an adverse reaction or the initial treatment did not provide relief) and settled on a different preventive treatment. Despite other possible reasons, the high discontinuation rate plus the additional observations of initial treatment with opioids or barbiturates and the excessive use of any 
acute treatment, indicate poor treatment management.

These results should also be viewed within the context of certain limitations associated with a retrospective administrative claims analysis. First, diagnostic codes are subject to coding/data entry error and lack of clinical specificity as they are for billing purposes only. Second, there is an inability to assess migraine severity in claims data due to the lack of clinical markers in these data. The evaluation of a link between utilization and treatment patterns with migraine severity among new migraine patients using survey or medical chart data is a worthwhile future study. Third, as our study relied on pharmacy claims for reporting medication use and identifying patients with migraine, these claims may not reflect actual use and do not account for over-the-counter medications. As such, patients may have filled their prescription and then pursued a non-pharmacological treatment and stopped their pharmacologic treatment [20, 21]. Fourth, this study relied on available data in MarketScan, which may not reflect all of a patient's medical history; as such, some patients may actually be prevalent migraine patients and not newly diagnosed. Also, this study had a limited follow-up time. There is a potential time lag from the original migraine diagnosis by a primary care or ER physician to a referral to a neurologist, as patients may have been treated successfully and therefore not need the referral or they may have pursued other reasons for their migraine diagnosis before seeking a referral, both of which could result in an underestimation of people who are ultimately seen by a neurologist in this study [22]. Finally, this study only analyzed those patients newly diagnosed with migraine who were aged 18-64 and had commercial insurance; as such, the results may not be generalizable to those migraine patients without insurance plans, with different insurance plans or who are older.

\section{CONCLUSIONS}

This analysis of treatment patterns of newly diagnosed migraine patients suggests poor migraine management characterized by inadequate preventive treatment persistence, initial treatment with opioids and barbiturates instead of less potent acute medications and excessive use of acute medications (particularly opioids and barbiturates). Information on prescriber type was unavailable; therefore, it is possible that practices vary among different types of physicians treating migraine. As migraine severity could not be evaluated, these treatment patterns may also reflect changes in migraine severity. Additional studies evaluating treatment patterns and HRU, particularly new referrals to neurologists or other headache specialists, among newly diagnosed migraine patients is warranted to further understand migraine management among those newly diagnosed and provide insight into improving initial management strategies among migraine patients. In addition to studies evaluating pharmacologic management of migraine, studies evaluating non-pharmacologic management of migraine are warranted.

\section{ACKNOWLEDGEMENTS}

Funding. Sponsorship for this study and Rapid Service Fee were funded by Amgen, Inc. (Thousand Oaks, CA, USA). All authors had full access to all of the data in this study and take complete responsibility for its integrity as a whole.

Authorship. All named authors meet the International Committee of Medical Journal Editors (ICMJE) criteria for authorship for this article, take responsibility for the integrity of the work as a whole, and have given their approval for this version to be published. Machaon Bonafede and Desai Pooja conceived and designed the project. Machaon Bonafede, Donna McMorrow and Virginia Noxon analyzed the data. Donna McMorrow and Virginia Noxon wrote the manuscript. All authors interpreted data, revised and commented on the manuscript and approved the final version. 
Disclosures. Pooja Desai, and Sandhya Sapra are employees of Amgen, Inc. and own Amgen stock. Machaon Bonafede, Donna McMorrow and Virginia Noxon are employees of IBM Watson Health, which received a research contract to conduct this study with and on behalf of Amgen, Inc. Stephen Silberstein is employee of Jefferson Medical College, and reports receipt of consulting fees from Alder Biopharmaceuticals; Allergan, Inc.; Amgen; Avanir Pharmaceuticals, Inc.; Curelator, Inc.; Dr. Reddy's Laboratories; eNeura Inc.; electroCore Medical, LLC; Lilly USA, LLC; Medscape, LLC.; NINDS; Supernus Pharmaceuticals, Inc.; Teva Pharmaceuticals; Theranica; and Trigemina, Inc.

Compliance with Ethics Guidelines. This article is based on previously conducted studies and does not contain any studies with human participants or animals performed by any of the authors. All database records are statistically deidentified and certified to be fully compliant with US patient confidentiality requirements set forth in the Health Insurance Portability and Accountability Act of 1996. Because this study used only de-identified patient records and did not involve the collection, use, or transmittal of individually identifiable data, Institutional Review Board approval to conduct this study was not required.

Data Availability. The datasets generated analyzed during the current study are not publicly available as they were used under license from IBM Watson Health but are available from the corresponding author on reasonable request and with permission of IBM Watson Health.

Open Access. This article is distributed under the terms of the Creative Commons Attribution-NonCommercial 4.0 International License (http://creativecommons.org/licenses/ by-nc/4.0/), which permits any noncommercial use, distribution, and reproduction in any medium, provided you give appropriate credit to the original author(s) and the source, provide a link to the Creative Commons license, and indicate if changes were made.

\section{REFERENCES}

1. Headache Classification Subcommittee of the International Headache Society. The international classification of headache disorders, 2nd edition. Cephalalgia. 2004;24[Suppl 1]:9-160.

2. Lipton RB, Stewart WF, Diamond S, Diamond ML, Reed M. Prevalence and burden of migraine in the United States: data from the American Migraine Study II. Headache. 2001;41(7):646-57.

3. Silberstein SD. Practice parameter: evidence-based guidelines for migraine headache (an evidencebased review): report of the Quality Standards Subcommittee of the American Academy of Neurology. Neurology. 2000;55(6):754-62.

4. American Migraine Foundation. Preventive treatments. 2016. https://americanmigrainefoundation. org/understanding-migraine/preventive-treatments/. Accessed 4 Feb 2020.

5. Institute for Clinical and Economic Review (ICER). Calcitonin gene-related peptide (CGRP) inhibitors as preventive treatments for patients with episodic or chronic migraine: effectiveness and value. California Technology Assessment Forum: Institute for Clinical and Economic Review; 2018.

6. Urits I, Jones MR, Gress $\mathrm{K}$, et al. CGRP antagonists for the treatment of chronic migraines: a comprehensive review. Curr Pain Headache Rep. 2019;23(5):29.

7. Lipton RB, Bigal ME, Diamond $\mathrm{M}$, et al. Migraine prevalence, disease burden, and the need for preventive therapy. Neurology. 2007;68(5):343-9.

8. Viticchi G, Falsetti L, Bartolini M, et al. Migraine: incorrect self-management for a disabling disease. Neurol Int. 2018;10(1):7510.

9. Bonafede $\mathrm{M}$, Cai $\mathrm{Q}$, Cappell $\mathrm{K}$, et al. Factors associated with direct health care costs among patients with migraine. J Manag Care Spec Pharm. 2017;23(11):1169-76.

10. Freitag FG, Lyss H, Nissan GR. Migraine disability, healthcare utilization, and expenditures following treatment in a tertiary headache center. Bayl Univ Med Cent Proc. 2013;26(4):363-7.

11. Wu J, Hughes MD, Hudson MF, Wagner PJ. Antimigraine medication use and associated health care costs in employed patients. J Headache Pain. 2012;13(2):121-7.

12. American Academy of Neurology. Axon registry. 2016. https://www.aan.com/practice/axon-registry. Accessed 4 Feb 2020. 
13. American Migraine Foundation. American Registry for Migraine Research. 2016. https://american migrainefoundation.org/about-us/armr/. Accessed 4 Feb 2020.

14. Deyo RA, Cherkin DC, Ciol MA. Adapting a clinical comorbidity index for use with ICD-9-CM administrative databases. J Clin Epidemiol. 1992;45(6): 613-9.

15. Ong JJY, De Felice M. Migraine treatment: current acute medications and their potential mechanisms of action. Neurotherapeutics. 2018;15(2):274-90.

16. Miller S. The acute and preventative treatment of episodic migraine. Ann Indian Acad Neurol. 2012;15[Suppl 1]:S33-9.

17. Weatherall MW. The diagnosis and treatment of chronic migraine. Ther Adv Chronic Dis. 2015;6(3): 115-23.

18. Messali A, Owens G, Bloudek L, Kori S, Cole A, Chia J. Health care resource utilization following initiation of a triptan: a retrospective claims analysis. J Manag Care Spec Pharm. 2014;20(4):368-75.

19. Etemad LRea. Costs and utilization of triptan users who receive drug prophylaxis for migraine versus triptan users who do not receive drug prophylaxis. J Manag Care Pharm. 2005;11(2):137-44.

20. Peres MFP, Swerts DB, de Oliveira AB, Silva-Neto RP. Migraine patients' journey until a tertiary headache center: an observational study. J Headache Pain. 2019;20(1):88.

21. Viswanath O, Rasekhi R, Suthar R, Jones MR, Peck J, Kaye AD. Novel interventional nonopioid therapies in headache management. Curr Pain Headache Rep. 2018;22(4):29.

22. Davies PTG, Lane RJM, Astbury T, Fontebasso M, Murphy J, Matharu M. The long and winding road: the journey taken by headache sufferers in search of help. Prim Health Care Res Dev. 2019;20:e4. 